\title{
Al+AR: Cultural Heritage, Museum Institutions, Plastic Models and Prototyping. A State of Art
}

\author{
Marco Vitali \\ Giulia Bertola \\ Fabrizio Natta \\ Francesca Ronco
}

\section{Abstract}

The links between representation and artificial intelligence (Al) invade many fields of architectural research, recording continuous and significant advances: they require, on the one hand, a constant update of the state of the art and, on the other hand, careful consideration of the role of Representation in interdisciplinary research in this field.

The present contribution intends to investigate these intertwining in some of the most frequented research fields in recent years: the valorization and communication of Cultural Heritage and cultural tourism, the experiences in the museum field, the research on the role of the prototype within the processes of artificial intelligence applied to architecture.

Keywords

cultural heritage, museum, digital fabrication, artificial intelligence, augmented reality.

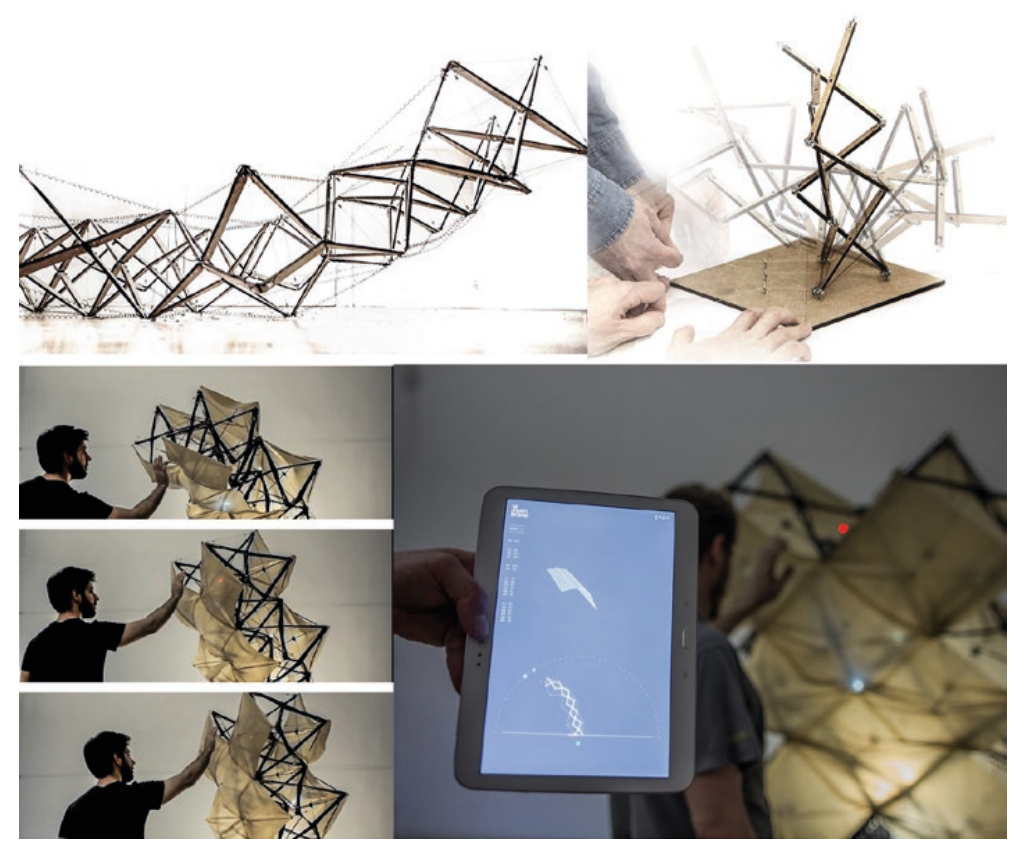




\section{Introduction (MV)}

The intertwining of representation - in particular in its manifestations in the field of augmented reality (AR) - with artificial intelligence (Al) spans many fields of architectural research and registers in the contemporary world continuous and significant advances that require, on the one hand, a constant updating of the state of the art and, on the other hand, careful considerations on the role of Representation in interdisciplinary research in this field. The present contribution intends to investigate these connections in some of the most frequented fields in architectural research in recent years: research on the enhancement and communication of Cultural Heritage and cultural tourism, research on the most recent and innovative experiences in the museum field, research on the role of the prototype within the processes of artificial intelligence applied to architecture.

According to the most recent experiences conducted on the valorization and communication of Cultural Heritage, the most used tools to ensure its success range from the creation of cognitive maps to the development of Al technologies for the automatic digitization of cultural heritage, or the use of Deep Learning for the recognition of monuments and the construction of mobile apps.

In the museum field, artificial intelligence is increasingly used to develop different tools such as robots, chatbots, and websites, which allow analyzing data related to visitors and collections, where the contribution of representation disciplines is nowadays mainly connected to the possible outputs of Object recognition operations and the applications of this technology. The link between plastic model, Al, and AR is articulated in several aspects based on the "human-model' interaction and is articulated in some prevalent research strands, such as studies on computational design methods developed for 3D printing and component evaluation, or the work on Responsive Architecture, in which the physical model is the medium through which to experiment and communicate the design of dynamic and adaptive buildings.

\section{$\mathrm{Al}$ (and AR) in Cultural Heritage (FN)}

According to the most recent experiences with the valorization and communication of Cultural Heritage $(\mathrm{CH})$, they should be the result of a balanced synergy between interaction, experience, and representation.

The current scenario offers research methodologies investigating Cultural Heritage through Artificial Intelligence (Al) tools to increasingly democratize the access of $\mathrm{CH}$, the development of Al technologies for automatic digitization, or the use of Deep Learning (DL) for monument recognition and the development of mobile apps. As an example, the study by Pisoni et al. (202I) proposes the use of $\mathrm{Al}$ to support the accessible design of $\mathrm{CH}$. One of the points of greatest research interest is now on eXplainable Al (XAl) techniques, "a suite of machine learning techniques that produces more explainable models while maintaining a high level of learning performance (e.g., prediction accuracy), and enable humans to understand'"[Barredo et al. 2020, p. 2]. The tools offered by these developments in technology can enable museums and $\mathrm{CH}$ sites to modify their knowledge transmission. By opening up informal learning opportunities to the general public, based on experience and personal interaction with $\mathrm{CH}$, gaps in Natural Language Processing (NLP) and ComputerVision (CV) can be assessed.

Another research approach is that by Traviglia and Del Bue (whose study focuses on the development of $\mathrm{Al}$ technologies for the automatic digitization of $\mathrm{CH}$ : the different nature of the $\mathrm{CH}$ assets does not facilitate the creation of common standards and protocols going in this direction. The main idea of the approach is to incrementally build a 3D reconstruction that is not metric (i.e. where positions are measurable with a metric reference) but rather in the space of projective geometry. In this way, the digitization is made user-independent making total automation of the scanning process possible [Traviglia, Del Bue 2019].

A line of research to be highlighted is that developed by Valerio Palma. He introduces a project aimed at studying the techniques of convolutional neural networks (CNN) in the field of architectural heritage, which workflow has still to be developed. The first steps and results in the development of mobile applications for monument recognition are discussed: 
while $\mathrm{Al}$ is just beginning to interact with the built environment through mobile devices, heritage technologies have long produced and explored digital models and spatial archives. The interaction between DL algorithms and state-of-art information modeling is addressed as an opportunity both to exploit heritage collections and to optimize new object recognition techniques [Palma 2019, pp. 55 I-556].

The developments that these research can bring are almost all aimed at enriching the databases, which are still quantitatively limited and refer much towards two-dimensional objects that are "easier" to read in ML algorithms. Another challenge related to Al relates to the use of personal data. The implementations that augmented $\mathrm{CH}$ experiences can provide necessarily involve reading and constantly updating regulations and ethical guides for the responsible use of this powerful tool.

\section{The Digital Transformation in Museums: Al and AR as Tools to Engage Visitors (FR)}

Within museum institutions, artificial intelligence is increasingly used to develop different tools such as robots, chatbots, and websites, which allow analyzing data related to visitors and collections [Styx, 2020]. The network "Museums + Artificial Intelligence"(n.d), founded in 2019 by Oonagh Murphy and Elena Villaespesa is proof of this growing interest in Al tools applied in this field. At the same time, $V R$ and $A R$ have become a popular trend worldwide for the dissemination, communication, and enhancement of cultural heritage [Bekele et al. 20 I 8].

The contribution of representation disciplines in the AR field is quite evident: just think of the use of 3D virtual models, holograms, graphics for specific apps, and gaming, up to True AR technology [Sandor 20 I5].The group coordinated by Geronikolakis (2020) proposes an interesting application in the field of cultural heritage conservation to improve the experience of visitors, involved in the construction and restoration of archaeological sites.

In the field of Al, the representation disciplines could mainly be involved in the outputs of Object Recognition operations. The applications of this technology involve the user also outside the museum, as in the project 'Recognition', winner in 2016 of the IK award [Styx, 2020] or the application 'Art Selfie'. Several museums base the exploration of their collections on Object Recognition: on color similarities, [Cooper Hewitt Smithsonian Design Museum, n.d; Dallas Art Museum, n.d.], formal, line direction, or space and light [Barnes Foundation, n.d].

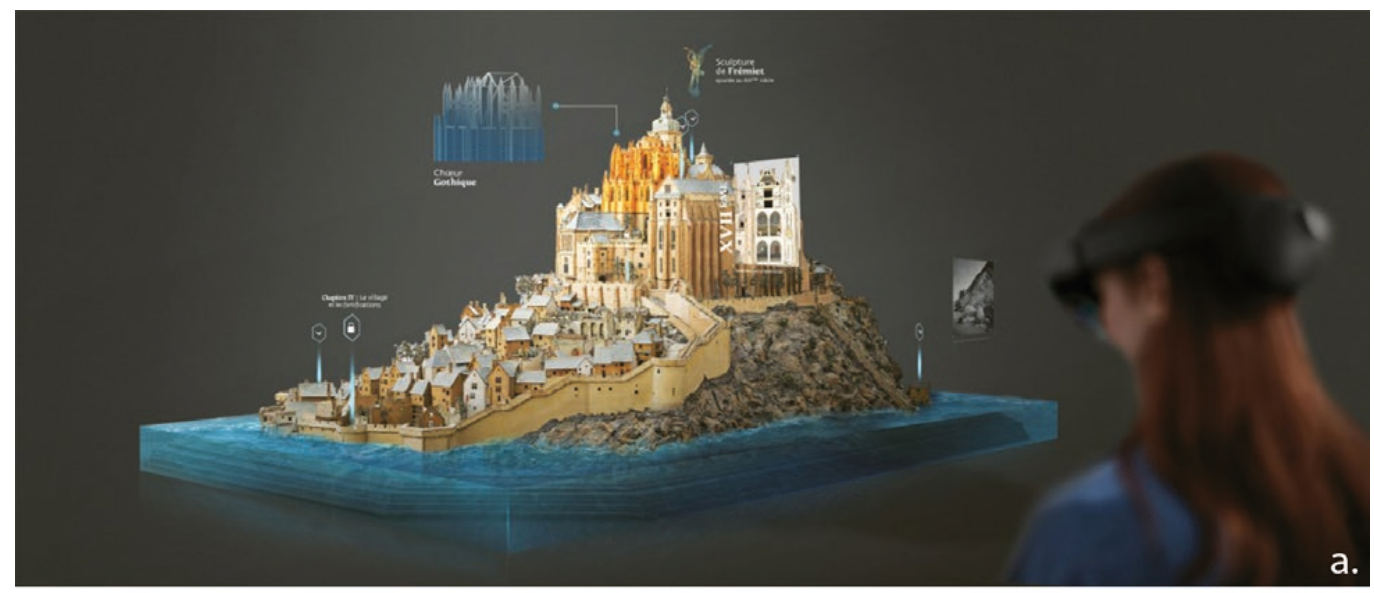

Fig. Ia. Exibition Mont Saint-Michel. Digital Perspectives on the Model (photo by Microsoft, source: https:// iciseattle.com/en/montsaint-michel-in-mohaif); Fig. Ib. The system of ICAR - CNR group ICAR-CNR group experienced in art [Caggianese et al., 2020]
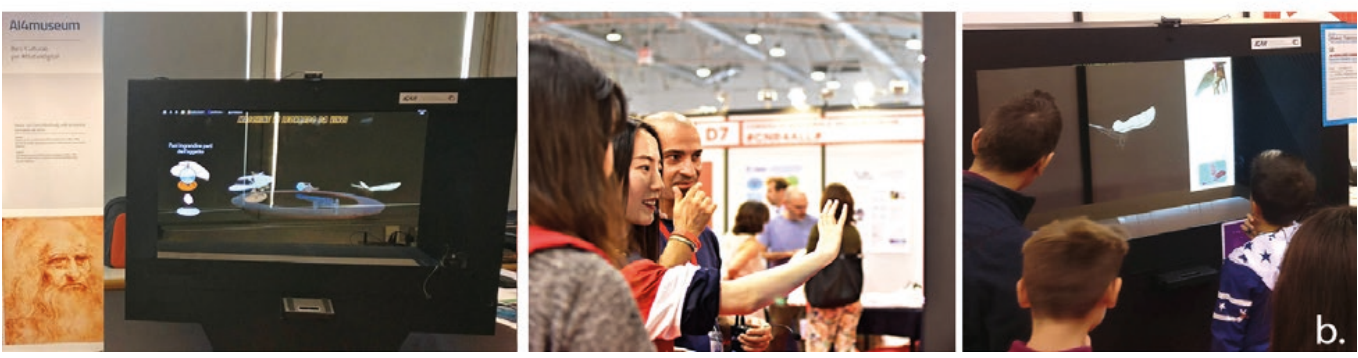
In recent years, technologies have made considerable progress in the creation of content tailored to the needs and interests of different types of users, with a democratic and humancentered approach. However, there are still few examples where Al has been used to foster accessibility and inclusion in the field of museums and cultural heritage [Pisoni et al., 202I].

The Al context is certainly characterized by a high level of multidisciplinarity. The mixed-use of Al/AR/VR technologies represents an important field of investigation to meet the needs of an increasingly wide audience, from an inclusive perspective.

The exhibition "Mont Saint-Michel - Digital Perspectives on the Model" (fig. I a), held in late 2019 at MOHAI (Museum of History and Industry) in Seattle, represents an example of the application of mixed techniques [Ici Seattle, 2019]. Visitors were immersed in the history of Mont-Saint-Michel by framing a real scale historical model of the site with a mixed reality device (Microsoft HoloLens 2).

Another example in this frame is the work conducted by ICAR-CNR [Caggianese et al,, 2020] related to the display of Leonardo da Vinci's machines (fig. I b). This presents the combined use of cutting-edge technologies, such as holograms, with artificial intelligence (Al) to offer the visitor an augmented space involving new forms of interaction (visualization, manipulation, and conversation).

\section{Al in Digital Fabrication Experiences on Architecture (GB)}

The link between real model, virtual reality and augmented reality is articulated on two main lines of research. The first one is based on the construction of informative real models, the second one on the interaction 'human-material' through the practices of 'augmented craftsmanship' and 'design by making'. Regarding the first, many studies are now focused on the creation of prototypes aimed at the transmission of knowledge and information of architectural heritage. In the last years, this has happened both through the realization of static models on which to apply immersive technologies, and with more complex dynamic systems in which physical and digital models have come to overlap to the point of almost confusing.

With regard to the application of immersive technologies, reference can be made to the experience carried out for the Basilica of Loreto [Rossi et al. 20 I7, pp. 239-255]. A project that involved the setting up of a space consisting of a multimedia table with a 3D printed physical model, conceived as a 'wunderkammer' equipped with a series of technological devices referable to different applications characterized by different levels of interactivity and immersiveness. In recent years, however, rapid advances in technology have begun to challenge even those aspects that go beyond the mere static and physical representation of architecture. An example is the interactive model of InFORM [Follmer et al. 20I3, pp. 4I7-426] a dynamic shape display proposed by the Massachusetts Institute of Technology (MIT), that suggests different ways of physical interaction in real time between users, physical model and digital data. Regarding the 'human-material' interaction, the main fields of investigation concern the application of biological principles on architectural construction and the study of innovative structural morphologies through digital fabrication methods. In particular, the ICD/ ITKE Research Pavilions are a series of full-scale prototypes realized through the integration of computational engineering, advanced analytical techniques and digital fabrication aimed at investigating the architectural potential of fiber composites [Doerstelmann et al. 20 I5]. Regarding the same themes, in London there are also the Protohouse project and the Flow-

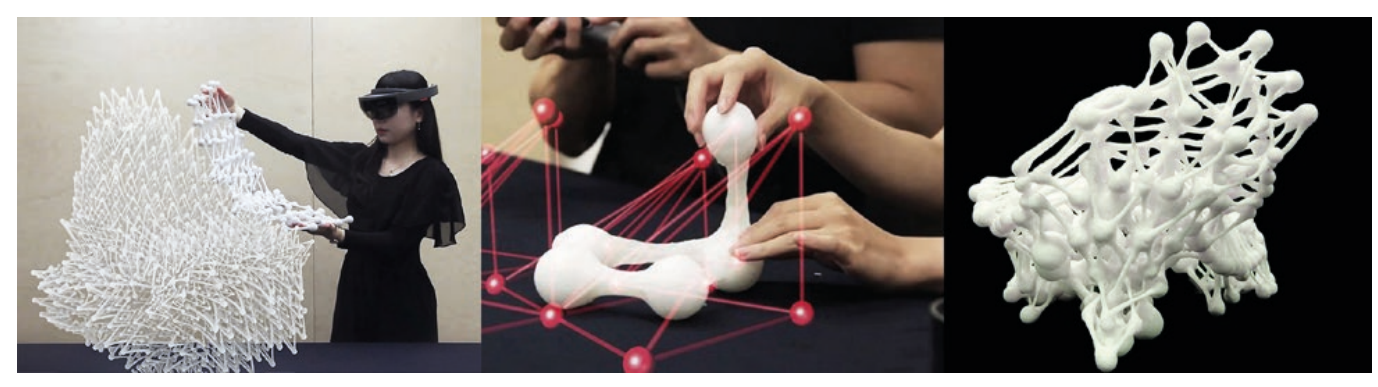


Morph project [Hahm et al. 2019, pp. 553-562] (fig. 2), that proposes an unconventional method of fabrication using Mixed Reality to materialize highly complex geometries that could not be realized manually or by robotic fabrication alone.

The practices of digitally augmented craftsmanship open to a series of reflections on the theme of interactive digital simulations and their link with traditional craft practices. Mario Carpo, within his book The Second Digital Turn [Carpo, 2017], emphasizes the value of such simulations as they allow today's artisans to learn intuitively, through mistakes, trial and attempts, by making and unmaking as many test samples as possible.

\section{References}

Barnes Foundation. Barnes Collection Online. https://collection.barnesfoundation.org/ (I March 202I).

Barredo Arrieta Alejandro et al. (2020). Explainable Artificial Intelligence (XAI): Concepts, Taxonomies, Opportunities and Challenges toward Responsible Al. In Information Fusion, 58, pp. 82-I 15.

Bekele Mafkereseb Kassahun, Pierdicca Roberto, Frontoni Emanuele, Malinverni Eva, Gain James (20 I 8). A Survey of Augmented,Virtual, and Mixed Reality for Cultural Heritage. In Journal on Computing and Cultural Heritage, I I (2), art. 7.

Caggianese Giuseppe, De Pietro Giuseppe, Esposito Massimo, Gallo Luigi, Minutolo Aniello, Neroni Pietro (2020). Discovering Leonardo with artificial intelligence and holograms: A user study. In Pattern Recognition Letters, I 3 I, pp. 36 I-367.

Carpo Mario (2017). The Second Digital Turn. Design Beyond Intelligence. Cambridge (MA): MIT Press.

Cooper-Hewitt Smithsonian Design Museum. The Collection. https://collection.cooperhewitt.org/ (I March 202 I).

Doerstelmann Moritz, Knippers Jan, Koslowski Valentin, Menges Achim, Prado Marshall, Schieber Gundula, Vasey Lauren (2015). ICD/ITKE Research Pavilion 2014-15: Fibre Placement on a Pneumatic Body Based on a Water Spider Web. In Architectural Design, 85, pp. 60-65.

Follmer Sean Weston, Leithinger Daniel, Olwal Alex, Hogge Akimitsu, Ishii Hiroshi (20I3), inFORM: Dynamic Physical Affordances and Constraints through Shape and Object Actuation, In Proceedings of the 26th annual ACM symposium on User interface software and technology (UIST '13). New York: ACM Digital Library, pp. 4 I 7-426.

Geronikolakis Efstratios, Zikas Paul, Kateros Steve, Lydatakis Nick, Georgiou Stelios, Kentros Mike, Papagiannakis George. (2020). A True AR Authoring Tool for Interactive Virtual Museums, In Liarokapis Fotis, Voulodimos Athanasios, Doulamis Nikolaos, Doulamis Anastasios (eds.). Visual Computing for Cultural Heritage. Cham: Springer, pp. 225-242.

Hahm Soomeen, Maciel Abel, Sumitiomo Eri, Rodriguez Alvaro Lopez (2019). FLOWMORPH. Exploring the human-material interaction in digitally augmented craftsmanship. In Proceedings of the 24th International Conference of the Association for Computer-Aided Architectural Design Research in Asia (CAADRIA), Vol. I, Hong Kong, CAADRIA, pp. 553-562.

High Level Expert Group on Artificial Intelligence (2019). Ethics Guidelines for Trustworthy Al; Technical Report. European Commission. Ici Seattle (2019). The Mont-Saint-Michel in the MOHAl. https:/iciseattle.com/en/mont-saint-michel-in-mohai/ (I March 202 I).

Jun JiWon, llubia Josep Alcover, Silverio Matteo (20I5). Remembrane. Responsive Kinetic Structure. http://www.iaacblog.com/programs/remembrane/ (I 5 March 202I).

Palma Valerio (2019). Towards Deep Learning for Architecture: A Monument Recognition Mobile App. In The International Archives of the Photogrammetry, Remote Sensing and Spatial Information Sciences, Volume XLII-2/N9, pp. 55 I-556.

Pisoni Galena, Diaz-Rodrìguez Natalia, Gijlers Hannie, Tonolli Linda (2021). Human-Centred Artificial Intelligence for Designing Accessible Cultural Heritage. In Applied Sciences, II (2), p. 870.

Rossi Daniele, Meschini Alessandra, Feriozzi Ramona, Olivieri Alessandro (2017). Cose dell'altro mondo. La realtà virtuale immersiva per il patrimonio culturale. In Luigini Alessandro., Panciroli Chiara (eds.), Ambienti digitali per l'educazione all'arte e al patrimonio. Milano, FrancoAngeli Open Access, pp. 239-255.

Sandor Christian, Fuchs Martin, Cassinelli Alvaro, Li Hao, Newcombe Richard, Yamamoto Goshiro, Feiner Steven (20 I5). In Breaking the barriers to true augmented reality. arXiv preprint arXiv: I5 I 2.0547 I (I march 202 I).

Styx Lauren (2020). How are museums using artificial intelligence, and is Al the future of museums? Museum Next. https://www.museumnext.com/article/artificial-intelligence-and-the-future-of-museums/ (I march 202I).

Traviglia Arianna, Del Bue Alessio (2019). Intelligenza Artificiale per la Digitalizzazione 3D del Patrimonio Culturale, In ItalIA Italia Intelligenza Artificiale. https://www.google.com/search?client=firefox-b-d\&q=Traviglia+Arianna\%2C+Del+Bue+Alessio+\%282019\%29. + Intelligenza+Artificiale+per+la+Digitalizzazione+3D+del+Patrimonio+Culturale\%2C+In+ItalIA+Italia+Intelligenza+Artificiale (I march 202 I).

\section{Authors}

Marco Vitali, Dept. of Architecture and Design, Politecnico di Torino, marco.vitali@polito.it

Giulia Bertola, Dept. of Architecture and Design, Politecnico di Torino, giulia.bertola@polito.it

Fabrizio Natta, Dept. of Architecture and Design, Politecnico diTorino, fabrizio.natta@polito.it

Francesca Ronco, Dept.of Architecture and Design, Politecnico di Torino, francesca.ronco@polito.it 
